\title{
Enteral nutrition in geriatric patients
}

\author{
Yitshal N. Berner
}

Received: 5 August 2008 / Accepted: 8 December 2008 / Published online: 27 January 2009

(c) Springer-Verlag 2009

\begin{abstract}
The elderly are an important part of the population. They are more frail, more often sick and it takes them longer to get better; therefore they consume a larger proportion of health services. Nutrition is adjuvant to medical treatment. Swallowing disorders are common in elderly patients. These may be manifestations of different neurological disorders, but the most common swallowing disorder is deconditioning during acute illness. Enteral nutrition bypasses the swallowing system, using nasogastric tubes or percutaneous gastric and enteric tubes. These feeding technologies can supply the needs of the elderly patient. Nevertheless, rehabilitation of the swallowing system, giving the elderly patient optimal functionality, is our main task. In addition to the medical treatment, logotherapists also have a crucial role in swallowing rehabilitation. Enteral nutrition (EN) is an essential part of geriatric medicine.
\end{abstract}

Key words Elderly · Nutrition support · Enteral nutrition · Ethics
The ageing population and its special health problems

An increase of about 2.5 years per decade has been observed in the life span of Israelis during the second half of the 20th century. A similar trend has been found in other Western societies (e.g., 1.8 years per decade in the United States). Consequently, the elderly population (defined as 65 year old and older) is constantly growing, with elderly people over 85 year old representing the fastest growing segment. Morbidity prevalence in this specific age group is of greater magnitude. Nutritional status derived from the intake of different nutrients is one of the components determining the physiological, medical and functional states of the elderly. Nutritional status is assessed as part of the Comprehensive Geriatric Assessment (CGA) [1].

Most clinical professionals would agree that in the care of sick or frail elderly patients, nutritional and hydration concerns often rank far too low on the list of evaluation and treatment priorities. In hospitals and nursing homes [2, 3], and in the community, elderly patients often receive a variety of costly and complex medical treatments, e.g., extensive drug therapy [4] and mechanical ventilatory support [5], while routine provision of adequate food and fluids is neglected. Compared with the many serious maladies already established and diagnosed in elderly patients, being at risk of malnutrition sometimes seems less than urgent. The need for nutritional assessment and intervention is particularly crucial in this age group because of a higher incidence of chronic diseases and a myriad of socioeconomic factors that increase the likelihood of malnutrition [6]. Though this age group has particular needs, only relatively small-scale research has been conducted.

\author{
Y.N. Berner (西) \\ Geriatric Medicine, \\ Meir Medical Center, \\ Kfar Saba Sackler Faculty of Medicine, \\ Tel Aviv University, Israel \\ e-mail: ferrari_cl@hotmail.com
}




\section{Age-related physiological changes leading to nutritional} deficiencies

Weight loss may reflect changes in appetite, dentition, taste, depression, comorbidity, poverty, isolation, constipation and other factors. According to data collected for healthy non-smoking subjects aged 18-100 years, between the ages of 30 and 70 there is a continuous decline of about $0.1-1 \%$ (on average about $0.5 \%$ ) per year in the function of many tissues and organs [7], which is manifested in the shape, needs and metabolism of the older person. However, regardless of cause, loss of lean body mass is an inevitable consequence of this agerelated weight loss. Physical activity declines with age, especially in developed societies, depriving muscles of what is probably their most important environmental stimulus to maintain their mass and function, as was previously discussed.

Changes in the gastrointestinal system also affect nutritional status in the elderly. The dental status deteriorates, i.e., there is loss of teeth, the remaining teeth are unsteady and artificial dentures may be ill fitting and cause discomfort. The swallowing process is less synchronised due to changes in the pharyngeal structure and deterioration in its neural control because of a decrease in neural conductivity that affects neural response. The swallowing mechanism is affected in different neurological disorders like cerebrovascular accident (CVA), Parkinson's disease and others, and if medical and rehabilitation treatment cannot overcome it there is a need to bypass the swallowing system for feeding using a nasogastric tube or percutaneous gastrostomy, either endoscopic or surgical. In patients with dysphagia due to acute neurological damage secondary to CVA, the dysphagia is either reversible and benefits from rehabilitation treatment like any other temporary neurological damage, or remains as permanent stable damage. In this case bypassing the disturbed swallowing mechanism remains the only solution for feeding these patients. Nevertheless, these patients' neurological condition remains stable, in contrast to the dementia patients, whose dysphagia represents further continuous progression of their disease.

Another swallowing disorder takes place in patients with advanced dementia where the swallowing problem reflects advancement of the progressive disorder. An inadequate intake of energy and nutrients is a common problem in demented patients. Undernutrition may be caused by several factors including anorexia (common cause: polypharmacotherapy), insufficient oral intake (forgetting to eat), depression and apraxia of eating or, less often, enhanced energy requirement due to hyperactivity. In advanced stages of dementia, dysphagia may develop and might be an indication for enteral nutrition $(\mathrm{EN})$ in a few cases.

The most common swallowing disorder in the elderly is part of deconditioning after acute illness, which may be cured with the cure of the acute illness, rehabilitation of swallowing mechanism and adjuvant nutrition support. The correct diagnosis of the swallowing disorder and adequate treatment are in the best interest of the elderly patient.

\section{Protein and energy deficiencies in the elderly}

The elderly eat considerably smaller amounts of food and eat less often than younger adults. Especially at times of acute or chronic illness, this lower intake can lead to energy deficit and general malnutrition accompanied by deteriorated mood, a condition often defined as failure to thrive (FTT) [8]. Forty percent of elderly hospital admissions in the United Kingdom are undernourished, half severely so. In a recent study, Allison et al. [9] showed that elderly patients consume less than $70 \%$ of their energy (recommended intake, $30-35 \mathrm{kcal} / \mathrm{kg} /$ day) and protein (recommended intake, $1 \mathrm{~g} / \mathrm{kg} /$ day) requirements.

Hypoalbuminaemia is found in more than $60 \%$ of malnourished geriatric patients and albumin remains one of the most sensitive markers of malnutrition. Hypoalbuminaemia arises because diseases and multiple morbidity are frequent in the elderly and they regularly result in the release of cytokines, such as interleukin-1 (IL-1), IL-6 and tumour necrosis factor- $\alpha$ (TNF- $\alpha$ ). The cytokines initiate catabolic phase characterised by breakdown of muscle cells as well as rapid loss of appetite. Aversion to meat consumption in diseased elderly people is well known. Illness and lack of appetite preserve the catabolic state. This is a common phenomenon in geriatric patients. Lack of appetite and the specific cytokine pattern [10] lead to significantly decreased food consumption. Because the albumin deficit is hardly noticed at its early stage, or if noticed is not attributed to preexisting malnutrition [11], malnutrition persists and might even get worse after the patient's admission to the hospital [9]. Without prompt diagnosis and appropriate countermeasures, the patient's nutrition parameters will continue to deteriorate from day to day. In such a situation, refeeding to restore normal nutrition parameters can take days or weeks [12].

\section{Active enteral nutrition support}

Active nutritional support using the technique of tube feeding (TF) is a common practice in elderly patients with advanced dementia and other neurological disor- 
ders in Israel [13]. In 2008 there are about 1600 elderly patients receiving active enteral nutritional support through nasogastric tubes and another 800 through percutaneous endoscopic gastrostomy (PEG). Nutrition support is by definition adiuvant (or supports living efforts) to medical and surgical treatments. It has several levels of intervention, starting with dietary manipulation to improve quantity and quality of nutritional intake; swallowing rehabilitation; addition of specific nutrients in different forms like iron, other minerals and vitamins, energy and protein to the existing diet; and using artificial formulas of food containing all the nutritional ingredients in recommended amounts, defined as medical foods. When medical foods are delivered by TF, either nasogastric or gastrostomy and jejunostomy, bypassing the swallowing mechanism, nutrition support becomes active enteral feeding. Parenteral nutrition is the other form of nutrition support, delivering the nutritional component in an active form using intravenous solutions when gastrointestinal insufficiency exists to a certain degree and there is a need to bypass this system in order to supply the patient with sufficient nutrition.

Recently meta-analysis of protein and energy supplementation in older patients has provided the information that current evidence does not support routine supplementation at home or in any setting, except for undernourished elderly patients in the acute care setting [14]. This study strengthens the American College of Nutrition, National Institute of Health and the American Society of Parenteral and Enteral Nutrition conclusions from 1997 [15] that there are no published observations providing direct evidence that wasting is a cause of death and that reversal of wasting improves outcomes.

\section{Ethical considerations in decisions on active enteral nutrition support}

Some studies on oral nutrition support (ONS) have shown improvements in body weight [16-18]. In tubefed demented elderly patients, two studies reported weight gain $[19,20]$, but two others reported no change $[14,21]$. Available trials regarding the effects of ONS $[16,17]$ or $\mathrm{TF}[21,22]$ on functional status report no improvement. In terms of survival, most studies show no benefit [22-24]. On the other hand, Rudberg et al. [25] described lower mortality, compared to controls, at 30 days and 1 year in enterally fed patients with severe swallowing disorders including patients with cognitive impairment. Very low mortality rates have been reported in PEG-fed demented nursing home residents [26, 27]. In the Israeli study [27] differences have been shown between those with progressive dementia and the others. On the other hand, in one retrospective study comparing mortality rates in different diagnostic groups, the outcome was worst among the demented [28].

According to the European Society of Parenteral and Enteral Nutrition (ESPEN)'s study published in 2005 [29], dementia is the most controversial area regarding PEG placement. The stated aims of TF in advanced dementia included improving functional status, avoiding hunger, improving comfort, preventing nutritional decline and its consequences, preventing aspirations and reducing the incidence of pressure ulcers and infections.

Decisions on active enteral feeding, as any other medical treatment, have to be made according to the four principles of medical ethics as postulated in the Helsinki declaration: (1) patient's autonomy, (2) social justice, (3) beneficence and (4) non-maleficence. In order to consider these principles it is suggested to divide them into two groups: the first two are those which are not dependent on the physician, while the last two are dependent on the knowledge and experience of the physician and his evaluation capacities concerning each patient.

Since most demented patients who need active nutrition support are not able to express their wishes according to the first principle and society gives the option of active nutritional support to these patients, a decision has to be made according to our medical experience and knowledge using the two principles of patient's beneficence and non-maleficence. More specifically, the following questions must be asked:

Does the patient suffer from a condition that is likely to benefit from EN?

Will nutritional support improve outcome and/or accelerate recovery?

Does the patient suffer from an incurable disease, but one in which quality of life and well-being can be maintained or improved by EN?

Does the anticipated benefit outweigh the potential risks?

These questions are discussed in detail, answering specific questions concerning the evidence for beneficence and non-maleficence in ESPEN's guidelines for EN support in the elderly [30]. Nevertheless it remains the main task of the clinician to evaluate and decide if the treatment is adequate or not for each patient.

Conflict of interest statement The author declares that he has no conflict of interest related to the publication of this article.

\section{References}

1. Feldman J, Peleg L, Yaretzky A (1999) Clinical, social and economic aspects of comprehensive geriatric assessment. Harefua 136:933-935 
2. McWhirter JP, Pennington CR (1994) Incidence and recognition of malnutrition in hospital. BMJ 308:945-948

3. Morley JE, Silver AJ (1995) Nutritional issues in nursing home care. Ann Intern Med 123:850-859

4. Flaherty JH, Perry HM 3rd, Lynchard GS, Morley JE (2000) Polypharmacy and hospitalization among older home care patients. J Gerontol A Biol Sci Med Sci 55:M554-559

5. Montuclard L, Garrouste-Orgeas M, Timsit JF et al (2000) Outcome, functional autonomy, and quality of life of elderly patients with a long-term intensive care unit stay. Crit Care Med 28:3389-3395

6. Barrocas A, Belcher D, Champagne C, Jastram C (1995) Nutrition assessment: practical approaches. Clin Geriatr Med 11:675-713

7. Sehl ME, Yates FE (2001) Kinetics of human aging: I. Rates of senescence between ages 30 and 70 years in healthy people. J Gerontol 56A:B198-208

8. Palmar RM (1990) Failure to thrive in the elderly: diagnosis and management. Geriatrics 45:47-55

9. Allison SP, Rawlings J, Field J et al (2000) Nutrition in the elderly hospital patient Nottingham studies. J Nutr Health Aging 4:54-57

10. Bonnefoy M, Coulon L, Bienvenu J et al (1995) Implication of cytokines in the aggravation of malnutrition and hypercatabolism in elderly patients with severe pressure sores. Age Ageing 24:37-42

11. Pokrywka HS, Koffler KH, Remsburg R et al (1997) Accuracy of patient care staff in estimating and documenting meal intake of nursing home residents. J Am Geriatr Soc 45:1223-1229

12. Seiler WO (2001) Clinical pictures of malnutrition in ill elderly subjects. Nutrition 17:496-498

13. Dwolatzky T, Berezovski S, Friedmann R et al (2001) A prospective comparison of the use of nasogastric and percutaneous endoscopic gastrostomy tubes for long-term enteral feeding in older people. Clin Nutr 20:535-540

14. Milne AC, Avnell A, Potter J (2006) Meta analysis: protein and energy supplementation in older people. Ann Int Med 144:37-48

15. Klein S, Kinney J, Jeejeebhoy K et al (1997) Nutrition support in clinical practice: review of published data and recommendations for future research directions. Summary of a conference sponsored by the National Institutes of Health, American Society for Parenteral and Enteral Nutrition, and American Society for Clinical Nutrition. Am J Clin Nutr 66:683-691

16. Carver AD, Dobson AM (1995) Effects of dietary supplementation of elderly demented hospital residents. J Hum Nutr Diet $8: 389-394$
17. Wouters-Wesseling W, Wouters AE, Kleijer CN et al (2002) Study of the effect of a liquid nutrition supplement on the nutritional status of psycho-geriatric nursing home patients. Eur J Clin Nutr 56:245-251

18. Faxen-Irving G, Andren-Olsson B, af Geijerstam A et al (2002) The effect of nutritional intervention in elderly subjects residing in group-living for the demented. Eur J Clin Nutr 56:221-227

19. Peck A, Cohen CE, Mulvihill MN (1990) Long-term enteral feeding of aged demented nursing home patients. J Am Geriatr Soc 38:1195-1198

20. Golden A, Beber C, Weber R et al (1997) Long-term survival of elderly nursing home residents after percutaneous endoscopic gastrostomy for nutritional support. Nurs Home Med 5:382-389

21. Callahan CM, Haag KM, Weinberger M et al (2000) Outcomes of percutaneous endoscopic gastrostomy among older adults in a community setting. J Am Geriatr Soc 48:1048-1054

22. Nair S, Hertan H, Pitchumoni CS (2000) Hypoalbuminemia is a poor predictor of survival after percutaneous endoscopic gastrostomy in elderly patients with dementia. Am J Gastroenterol 95:133-136

23. Meier DE, Ahronheim JC, Morris J et al (2001) High short-term mortality in hospitalized patients with advanced dementia: lack of benefit of tube feeding. Arch Intern Med 161:594-599

24. Murphy LM, Lipman TO (2003) Percutaneous endoscopic gastrostomy does not prolong survival in patients with dementia. Arch Intern Med 163:1351-1353

25. Rudberg MA, Egleston BL, Grant MD, Brody JA (2000) Effectiveness of feeding tubes in nursing home residents with swallowing disorders. J Parent Ent Nutr 24:97-102

26. Mitchell SL, Kiely DK, Lipsitz LA (1997) The risk factors and impact on survival of feeding tube placement in nursing home residents with severe cognitive impairment. Arch Intern Med 157:327-332

27. Abuksis G, Mor M, Segal N et al (2000) Percutaneous endoscopic gastrostomy: high mortality rates in hospitalized patients [see comments]. Am J Gastroenterol 95:128-132

28. Skelly RH (2002) Are we using percutaneous gastrostomy properly in the elderly. Curr Opin Clin Nutr Care 5:35-42

29. Loesser Ch, Aschl G, Hebutrene X et al (20005) ESPEN Guidelines on artificial enteral nutrition - percutaneous endoscopic gastrostomy (PEG). Clin Nutr 24:848-861

30. Volkert D, Berner YN, Berry E et al (2006) ESPEN guidelines on enteral nutrition: geriatrics. Clin Nutr 25:330-360 